\title{
Regulation of Dendritic Spines, Spatial Memory, and Embryonic Development by the TANC Family of PSD-95-Interacting Proteins
}

\author{
Seungnam Han, ${ }^{1 *}$ Jungyong Nam, ${ }^{1 *}$ Yan Li, ${ }^{1 \star}$ Seho Kim, ${ }^{1}$ Suk-Hee Cho, ${ }^{2}$ Yi Sul Cho, ${ }^{3}$ So-Yeon Choi, ${ }^{4}$ Jeonghoon Choi, ${ }^{1}$ \\ Kihoon Han, ${ }^{1}$ Youngrim Kim, ${ }^{1}$ Moonseok Na, ${ }^{1}$ Hyun Kim,,${ }^{4}$ Yong Chul Bae, ${ }^{3}$ Se-Young Choi, ${ }^{2}$ and Eunjoon Kim ${ }^{1}$ \\ ${ }^{1}$ National Creative Research Initiative Center for Synaptogenesis and Department of Biological Sciences, Korea Advanced Institute of Science and \\ Technology, Daejeon 305-701, Korea, ${ }^{2}$ Department of Physiology and Dental Research Institute, Seoul National University School of Dentistry, Seoul \\ 110-749, Korea, ${ }^{3}$ Department of Anatomy and Neurobiology, Brain Korea 21, School of Dentistry, Kyungpook National University, Daegu 700-412, Korea, \\ and ${ }^{4}$ Department of Anatomy and Division of Brain Korea 21 Biomedical Science, College of Medicine, Korea University, Seoul 136-705, Korea
}

PSD-95 (postsynaptic density-95) is thought to play important roles in the regulation of dendritic spines and excitatory synapses, but the underlying mechanisms have not been fully elucidated. TANC1 is a PSD-95-interacting synaptic protein that contains multiple domains for protein-protein interactions but whose function is not well understood. In the present study, we provide evidence that TANC1 and its close relative TANC2 regulate dendritic spines and excitatory synapses. Overexpression of TANC1 and TANC2 in cultured neurons increases the density of dendritic spines and excitatory synapses in a manner that requires the PDZ (PSD-95/Dlg/Z0-1)-binding C termini of TANC proteins. TANC1-deficient mice exhibit reduced spine density in the CA3 region of the hippocampus, but not in the CA1 or dentate gyrus regions, and show impaired spatial memory. TANC2 deficiency, however, causes embryonic lethality. These results suggest that TANC1 is important for dendritic spine maintenance and spatial memory, and implicate TANC2 in embryonic development.

\section{Introduction}

PSD-95/SAP90 (postsynaptic density-95/synapse-associated protein 90), an abundant postsynaptic scaffolding protein at excitatory synapses, has been suggested to regulate dendritic spines and excitatory synapses through interaction with various postsynaptic density proteins (Funke et al., 2005; Montgomery et al., 2004; Fitzjohn et al., 2006; Okabe, 2007; Sheng and Hoogenraad, 2007; Keith and ElHusseini, 2008). However, the mechanisms underlying these regulatory functions have not been fully elucidated.

TANC1 was originally reported as a novel PSD-95-interacting protein (Suzuki et al., 2005). Because it contains several domains for protein-protein interactions, including ankyrin repeats, tetratricopeptide repeats (TPRs), a coiled-coil domain, and a C-terminal PDZ (PSD-95/Dlg/ZO-1) domain-binding motif, TANC1 is thought to function as a scaffold/adaptor protein. Potential Drosophila homologs of TANC1 termed Rolling pebbles regulate myoblast fusion in Drosophila (Menon and Chia, 2001;

Received June 17, 2010; revised Sept. 14, 2010; accepted Sept. 16, 2010.

This work was supported by the Korea Science Foundation grant (to S.Y.C.; 313-2007-2-C00630), the Basic Science Research Program (to Y.C.B.; R13-2008-009-01001-0), and the National Creative Research Initiative Program (to E.K.) from the Korean Ministry of Education, Science, and Technology. A part of this work was technically supported by the core facility service of the 21C Frontier Brain Research Center (M103KV010023-07K2201-02510). We thank the Kazusa DNA Research Institute for their generous gift of the KIAA clones (KIAA1728 and KIAA1636). We thank Dr. Pann-Ghill Suh (UNIST, Ulsan, Korea) for GRIP1 antibodies (43-8) and Dr. Yi-Ping Hsueh (Institute of Molecular Biology, Academia Sinica, Taipei, Taiwan) for CASK antibodies.

*S.H., J.N., and Y.L. contributed equally to the work.

Correspondence should be addressed to Eunjoon Kim, Department of Biological Sciences, Korea Advanced Institute of Science and Technology, Daejeon 305-701, Korea. E-mail: kime@kaist.ac.kr.

DOI:10.1523/JNEUROSCI.3128-10.2010

Copyright $\odot 2010$ the authors $\quad 0270-6474 / 10 / 3015102-11 \$ 15.00 / 0$
Rau et al., 2001). TANC1 mRNA in rats is expressed in various tissues, including the brain, where its expression is particular strong in the hippocampus (Suzuki et al., 2005). TANC1 proteins are localized at excitatory synapses, directly interact with PSD-95, and form a complex with diverse excitatory postsynaptic proteins including PSD-95, GKAP, AMPA receptors, NMDA receptors, and the $\alpha$ subunit of $\mathrm{Ca}^{2+} /$ calmodulin-dependent protein kinase II. More recently, TANC1 was shown to interact with MINK (Misshapen/NIKs-related kinase) and TNIK (Traf2- and Nckinteracting kinase), which belong to the STE20 family of mitogenactivated protein kinase kinase kinase kinases (MAP4Ks) (Nonaka et al., 2008). Interestingly, TANC1 is phosphorylated by MINK and TNIK, which act downstream of Rap2 (Taira et al., 2004; Nonaka et al., 2008), a Ras family small GTPase implicated in the negative regulation of dendritic spines and synaptic AMPA receptors (Pak et al., 2001; Pak and Sheng, 2003; Zhu et al., 2005; Fu et al., 2007; Ryu et al., 2008). Despite these interesting characteristics of TANC1 expressed in the brain, the functions of TANC proteins have not been reported.

In the present study, we identified and characterized TANC2, a close relative of TANC1. In addition, we explored the functions of TANC1 and TANC2 using cultured neurons and mouse genetic approaches. TANC1 and TANC2 overexpressed in cultured neurons have positive influences on the density of dendritic spines and excitatory synapses. Mice deficient in TANC1 exhibit a decrease in spine density in the $\mathrm{CA} 3$ region of the hippocampus, but not in the CA1 or DG regions. TANC1 knock-out mice also show impaired spatial learning. In contrast, a TANC2 deficiency causes embryonic lethality. These results implicate TANC family 
proteins in the regulation of dendritic spines, spatial learning, and embryonic development.

\section{Materials and Methods}

cDNA constructs. Human TANC1 [amino acids 217-1861; $\Delta \mathrm{C}$ (a mutant that lacks the last three residues), amino acids 217-1858) and human TANC2 (amino acids 129-1990; $\Delta$ C, amino acids 129-1897) were subcloned from the KIAA1728 and KIAA1636 into p3XFLAG-CMV (Sigma). Rat TANC1 (full length, amino acids 1-1849) was subcloned into pGW1 (British Biotechnology). For EGFP-tagged TANC2, human TANC2 (amino acids 129-1990) were subcloned into pEGFP-C1 (Clontech). The following expression constructs have been described: GW1PSD-95 (Kim et al., 1995) and GW1-PSD-95-EGFP (Arnold and Clapham, 1999). For yeast two-hybrid screen and assays, the PDZ2 domain of PSD-95 (amino acids 89-299) in the pBHA bait vector was used to screen a human brain yeast two-hybrid cDNA library contained in the pACT2 prey vector (Clontech). For yeast two-hybrid experiments, human TANC1 [wild-type (WT), amino acids 1661-1861; and $\Delta \mathrm{C}$, amino acids 1661-1858] were subcloned into pBHA. The PDZ domains in pGAD10 (a prey vector; Clontech) have been described previously (Choi et al., 2002, 2005). For shRNA TANC1 knockdown constructs, nucleotides 5499-5517 of rat TANC1 (CCA CCT CAC TTC AGC GAA A) was subcloned into pSuper.gfp/neo vector (OligoEngine). For TANC1 rescue experiments, human TANC1 (full length, amino acids 1-1861) was subcloned into pGW1.

Antibodies. Synthetic peptides mimicking the last 15 residues of TANC1 (LTAAKPKRSFIESNV; amino acids 1847-1861 of human TANC1) and TANC2 (AYQDNLYRQLSRDSR; amino acids 1957-1971 of rat TANC2) were used as immunogens to generate rabbit (1609) and guinea pig (1791) polyclonal antibodies, respectively. Rabbit polyclonal PSD-95 antibodies (1690) and guinea pig polyclonal SAP97 antibodies (1443) were generated by using H6-PSD-95 (amino acids 77-299 of human PSD-95) and GST-SAP97 (rat full length), respectively. Specific antibodies were affinity-purified using SulfoLink columns (Pierce). The specificity of the TANC antibodies (1609 and 1791) was determined by dot blot analysis. The following antibodies have been described; PSD-95 (HM319) (Kim et al., 1995), PSD-95 (SM55) (Choi et al., 2002), PSD-95 (1402) (Choi et al., 2005), PSD-93/chapsyn-110 (B9594) (Kim et al., 1996), SAP102 (1445) (Choi et al., 2005), EGFP (1167) (Choi et al., 2002), GRIP1 (43-8; a kind gift from Dr. Pann-Ghill Suh, UNIST, Ulsan, Korea) and CASK (a kind gift from Dr. Yi-Ping Hsueh, Institute of Molecular Biology, Academia Sinica, Taipei, Taiwan) (Hsueh and Sheng, 1999). The following antibodies were purchased; synaptophysin, MAP2, FLAG, $\alpha$-tubulin (Sigma), NeuN (Millipore Bioscience Research Reagents), and PSD-95 (Affinity Bioreagents).

In situ hybridization. In situ hybridization was performed as described previously (Han et al., 2009). Adult (6 weeks) rat brain sections (12 $\mu \mathrm{m}$ thick) were prepared with a cryostat (Leica CM 1950). The hybridization probes were prepared from pGEM7zf plasmids containing nucleotides 4621-5301 of rat TANC1 (XM_229944) and nucleotides 1931-2505 of rat TANC2 (XM_221028) using a Riboprobe System (Promega) and $\alpha-\left[{ }^{35} \mathrm{~S}\right] \mathrm{UTP}$.

Subcellular and PSD fractions. Subcellular fractionations of rat brains were prepared as described previously (Huttner et al., 1983). PSD fractions were purified as described previously (Cho et al., 1992). Immunoblot analysis of these fractions was performed using pan-TANC (1609), PSD-95 (SM55), and $\alpha$-tubulin antibodies.

In vivo pull down and coimmunoprecipitation assays. To generate GST fusion proteins for in vivo pull down assay, amino acids 1661-1861 and 1661-1858 of human TANC1 and amino acids 1790-1990 and 1790-1987 of human TANC2 were subcloned into pGEX4T-1 (GE Healthcare). For in vivo pull down, the crude synaptosomal fraction of adult rat brain was solubilized with DOC buffer ( $50 \mathrm{~mm}$ Tris- $\mathrm{HCl}, 1 \%$ sodium deoxycholate, $\mathrm{pH}$ 9.0), and dialyzed against binding/dialysis buffer (50 mм Tris- $\mathrm{HCl}$, $0.1 \%$ Triton X-100, pH 7.4). The supernatant was incubated with GSTTANC1 or GST-TANC2 (WT and $\Delta \mathrm{C}$ ). For in vivo coimmunoprecipitation, the crude synaptosomal fraction of adult rat brain (6 weeks) was solubilized with the RIPA buffer $(0.1 \%$ SDS, $0.5 \%$ DOC, $1 \%$ Triton $\mathrm{X}-100$ in PBS) and incubated with antibodies against TANC1/2 (1609, 20 $\mu \mathrm{g} / \mathrm{ml}$ ), PSD-95 (HM319, 1:250 or 1402, 1:100), PSD-93 (B9594, 1:250), SAP97 (1443, 1:250) and SAP102 (1445,1: 250). The precipitates were analyzed by immunoblotting with antibodies against TANC1/2 (1609), PSD-95 (SM55 or 1690), PSD-93 (B9594), SAP97 (1443), SAP102 (1445), GRIP1 (43-8) and CASK antibodies. For a more rigorous immunoprecipitation, RIPA lysates of the crude synaptosomal fraction were centrifuged at 50,000 rpm (TLA 100.2 rotor, Beckman) before immunoprecipitation.

Primary neuron culture, transfection, and immunohistochemistry. Cultured hippocampal neurons were prepared from embryonic (embryonic day 18) rat brain as described previously (Goslin and Banker, 1991). Cultures were plated on coverslips (Fisher Scientific) coated with polyD-lysine $(30 \mu \mathrm{g} / \mathrm{ml})$ and grown in Neurobasal medium (Invitrogen) supplemented with B27 (Invitrogen), $0.5 \mathrm{~mm}$ glutamine, and $12.5 \mu \mathrm{M}$ glutamate. To determine spine localization TANC proteins, neurons were transfected with FLAG-TANC1 or FLAG-TANC2 (WT or $\Delta \mathrm{C})+$ PSD-95-EGFP at days in vitro (DIV) 18 using the calcium phosphate method and stained at DIV 19 by triple immunofluorescence staining with TANC, EGFP, and synaptophysin antibodies. To determine the effects of TANC1 and TANC2 overexpression on dendritic spines and excitatory synapses, neurons at DIV 8 were transfected with FLAG-TANC1/2 expression constructs and visualized at DIV 15 by double immunofluorescence staining with FLAG and EGFP antibodies. For staining of brain slices with pan-TANC and TANC2 antibodies, the TSA Cyanine 3 system (PerkinElmer Life Sciences) was used for signal amplification.

Image acquisition and quantification. Fluorescent images were acquired by confocal microscopy (LSM510) using the same parameter settings for all scans. Each experiment was repeated three to five times, and neuronal images for analysis were randomly selected. Morphometric measurements were performed in a blinded manner using MetaMorph image analysis software (Universal Imaging). The synaptic localization of TANC proteins was determined by comparing the immunofluorescence intensity of TANC proteins in a PSD-95- and synaptophysin-positive spine to that of an adjacent dendritic trunk. The spine/dendrite intensity ratios of TANC and PSD-95 proteins were obtained from a total of $\sim 400$ measurements (15-20 neurons and $\sim 20$ spines/trunks per neuron). For the analysis of spine density in cultured neurons, spines were defined as dendritic protrusions of $0.5-3 \mu \mathrm{m}$ in length, with or without a head. Spine density was measured by counting the number of spines in 10-12 neurons ( $\sim 100 \mu \mathrm{m}$ total dendritic length per neuron). Spine dimensions were determined by measuring $\sim 300-500$ spines in $10-12$ neurons for each condition. The length of each spine was measured by manually drawing a vertical line from the base of the neck to the furthest point on the spine head. Spine width was measured by drawing a maximal line perpendicular to the length line of a spine. The density and dimensions of spines from a single neuron were grouped and averaged; means from multiple individual neurons were averaged to obtain a population mean and SEM. Spines in apical dendrites of the principal neurons in hippocampal subfields were used for spine analysis of DiOlistically labeled brain slices. In the case of CA3 pyramidal neurons, spines in distal dendrites beyond the thorny excrescences were analyzed. Statistical significance was determined by Student's $t$ test or ANOVA, as appropriate.

Electron microscopy. Hippocampal sections (60 $\mu \mathrm{m}$ thick) from 10 week-old mice were incubated overnight with pan-TANC antibodies (1609). For immunoperoxidase staining, sections were further incubated with biotinylated secondary antibodies for $2 \mathrm{~h}$ and ExtrAvidin peroxidase (Sigma) for $1 \mathrm{~h}$. The immunoperoxidase activity was revealed by nickel intensified 3,3'-diaminobenzidine tetrahydrochloride. For immunogold labeling, gold-conjugated secondary antibodies (1 nm gold, Aurion) were used, and the gold particles were intensified with silver enhancement kit (GE Healthcare). The area containing the stratum radiatum of the hippocampal CA1 region was trimmed. Images on a Hitachi H 7500 electron microscope (Hitachi) were captured by a MultiScan cooled CCD camera (ES1000W; Gatan) driven by Digital Montage software.

Electrophysiology. For field EPSP (fEPSP) recordings, baseline responses were collected at $0.07 \mathrm{~Hz}$ with a stimulation intensity that yielded a half-maximal response. Long-term potentiation in 6-week-old hippocampus was induced by four episodes of theta burst stimulation with $10 \mathrm{~s}$ intervals. TBS consisted of 10 stimulus trains delivered at $5 \mathrm{~Hz}$; each 

A

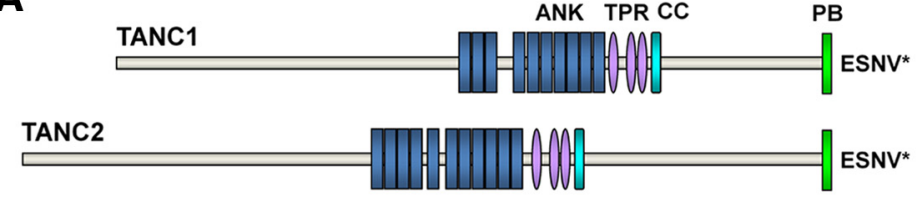

B

WT
$\beta$-Gal HIS3

PSD-95 PDZ 1-2 $++++++\quad-$

PSD-95 PDZ 3

PSD-93 PDZ 1-2 ++ $+{ }^{+} \quad-$

PSD-93 PDZ1-2 ++ ++

PSD-93 PDZ 3

SAP102 PDZ1-2 ++

SAP102 PDZ 3

GIP1 PDZ 4-6

pGAD10 alone
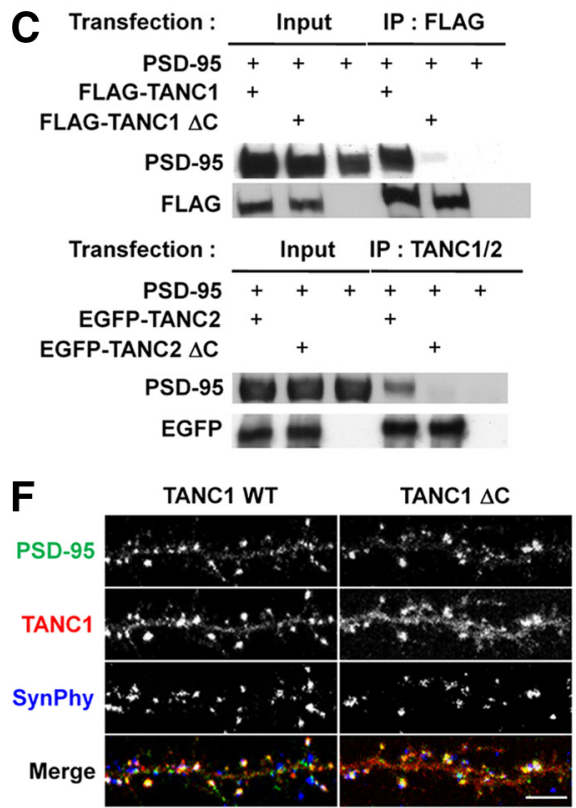
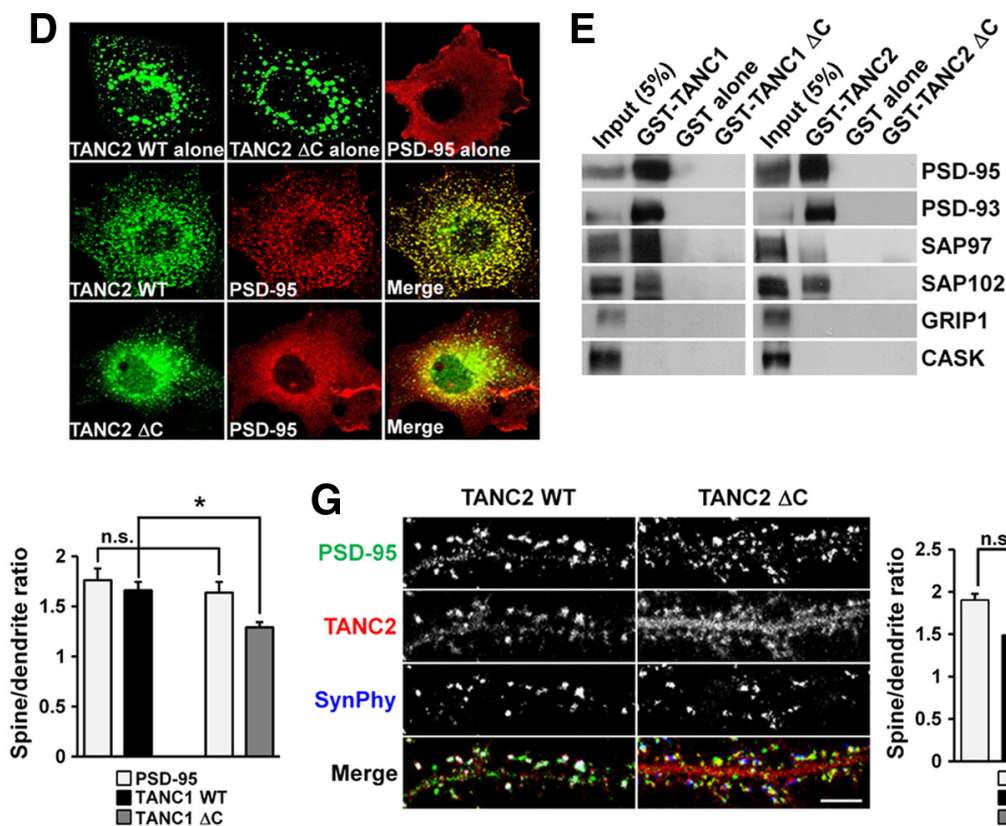

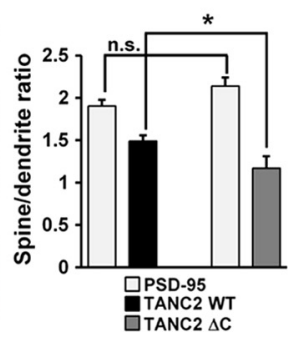

Figure 1. TANC1 and TANC2 interact with PSD-95 and are localized to dendritic spines in a manner requiring the PDZ-binding C-terminal motifs. A, Domain structure of TANC1 and TANC2. ANK, ankyrin repeats; TPR, tetratricopeptide repeats; CC, coiled-coil domain; PB, PDZ-binding motif that ends with the ESNV* sequence. B, TANC1 interacts with the PDZ domains from PSD-95 family proteins (PSD-95, PSD-93/chapsyn-110, and SAP102) but not with those from GRIP1 (a control PDZ protein) in yeast two-hybrid assays. pGAD10 alone, empty prey vector. HIS3 activity: $+++(>60 \%),++(30 \sim 60 \%)$, $+(10 \sim 30 \%),-$ (no significant growth); $\beta$-gal: $+++(<45 \mathrm{~min}),++(45 \sim 90 \mathrm{~min}),+(90 \sim 240 \mathrm{~min}),-$ (no significant $\beta$-galactosidase activity). C, TANC1 and TANC2 form a complex with PSD-95 in heterologous cells. HEK293T cell lysates doubly transfected with FLAG-tagged TANC1 (WT and $\Delta C$ ), or EGFP-tagged TANC2, and PSD-95 were immunoprecipitated (IP) with FLAG (or pan-TANC) antibodies and immunoblotted with the indicated antibodies. D, TANC2 coclusters with PSD-95 in heterologous cells in a manner requiring the PDZ-dependent interaction. COS cells were singly transfected with EGFP-tagged TANC2 (WT or $\Delta C$ ) or PSD-95, or doubly with EGFP-tagged TANC2 (WT or $\Delta C$ ) and PSD-95, and visualized by immunofluorescence staining with EGFP and PSD-95 antibodies. E, GST fusion proteins of TANC1 and TANC2 pull down PSD-95 family proteins (PSD-95, PSD-93, SAP97, and SAP102) but not other PDZ domain proteins from brain lysates. Detergentlysates of the crude synaptosomal fraction of adult rat brains were pulled down by GST fusion proteins containing the last 200 residues of TANC1 and TANC2 (WT and $\triangle C$ ) and immunoblotted with the indicated antibodies. F, G, TANC1 and TANC2 are localized to dendritic spines in a manner requiring the PDZ-binding C-terminal motifs. Cultured hippocampal neurons were doubly transfected with TANC1/2 (WT and $\Delta C$ ) and PSD-95-EGFP (DIV 18 -19) and immunostained for TANC1/2, EGFP (PSD-95), and synaptophysin (SynPhy). Scale bars, $10 \mu \mathrm{m}$. The spine localization of TANC and PSD-95 proteins were analyzed quantitatively by comparing the intensity ratios of these proteins at a spine and an adjacent dendritic trunk. Data represent mean $\pm \operatorname{SEM}\left(\operatorname{TANC} 1 \mathrm{WT}, 1.7 \pm 0.1, n=140\right.$ spines; $\operatorname{TANC} 1 \Delta C, 1.3 \pm 0.1, n=132 ; \mathrm{TANC}$ WT, $1.5 \pm 0.1, n=126 ; \operatorname{TANC2} \Delta C, 1.2 \pm 0.1, n=117 ;{ }^{*} p<0.05$, Student's t test). NS, not significant.

train consisted of four pulses at $100 \mathrm{~Hz}$. Long-term depression was induced in 4-5-week-old hippocampal slices with 900 paired-pulse stimulation delivered at $1 \mathrm{~Hz}$. Average responses ( \pm SEM) were expressed as percentage of baseline response (at least $10 \mathrm{~min}$ of stable responses). The experimenters were blind to the genotypes of the mice.

DiOlistic spine labeling. Three-week-old brain slices $(150 \sim 200 \mu \mathrm{m})$ from male mice (WT and TANC1 ${ }^{-1-}$ ) perfused with paraformaldehyde were labeled in the DG, CA3, and CA1 regions of hippocampus by the ballistic delivery of the lipophilic dye DiI (1,1'-dioctadecyl-3,3,3',3'tetramethylindocarbocyanine perchlorate) (catalog \#D-282, Invitrogen) as described previously (Gan et al., 2000). Dye-delivered slices were incubated at room temperature overnight for dye diffusion before image acquisition.

Open-field, elevated plus maze, rotarod, and Morris water maze assays. The open-field assay was performed in a white open-square chamber. The elevated plus-maze consisted of two open arms, two closed arms, and a center area, elevated to a height of $50 \mathrm{~cm}$ above the floor. Mice were placed in the center area and allowed to explore the space for $5 \mathrm{~min}$. For the accelerating rotarod assay, mice were placed on a rotating rod at a slow speed $(\sim 4 \mathrm{rpm})$ for $30 \mathrm{~s}$. The speed was gradually increased from 4 to $40 \mathrm{rpm}$ over $5 \mathrm{~min}$ (one trial per day), and the latencies to fall from the rod were measured. In the Morris water maze test, $\mathrm{TANC1}^{-1-}$ mice at $3 \sim 6$ months were trained to find the hidden platform with four trials per day (intertrial interval, $15 \mathrm{~min}$ ). Mice that did not find the platform within $90 \mathrm{~s}$ were gently guided to the platform and allowed to remain on the platform for $15 \mathrm{~s}$. In the probe test performed $24 \mathrm{~h}$ after the last training session, mice were released to the center region of the water maze apparatus and allowed to swim for $60 \mathrm{~s}$ in the absence of the platform. The number of exact crossings and quadrant occupancy were analyzed using Noldus EthoVision software (Noldus Information Technology).

\section{Results}

TANC1 and TANC2 interact with PSD-95 and are localized to dendritic spines in a manner requiring the PDZ-binding C-terminal motifs

Through database searches, we identified TANC2 as an additional member of the TANC family. TANC1 (1861 aa) and TANC2 (1990 aa) share $\sim 49.6 \%$ amino acid sequence identity and have an essentially identical domain structure, including the PDZ-binding motif at the $\mathrm{C}$ terminus (Fig. $1 A$ ). 
A

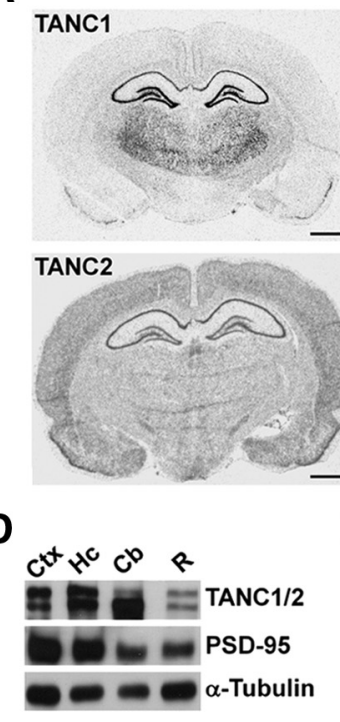

B

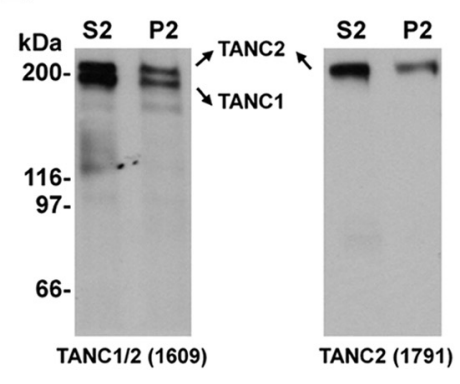

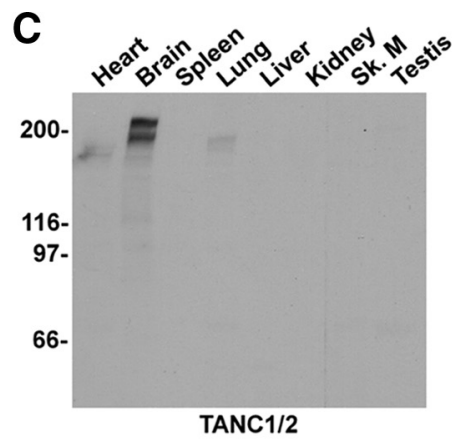

H

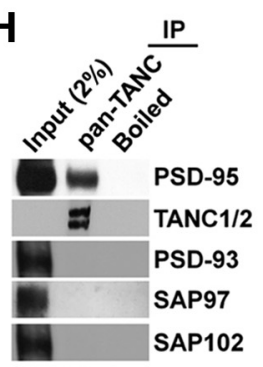

I

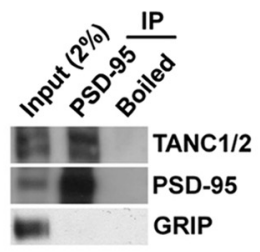

J

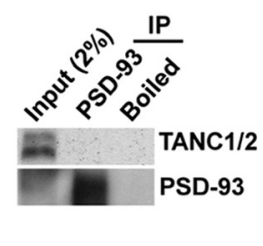

$\mathbf{F}$

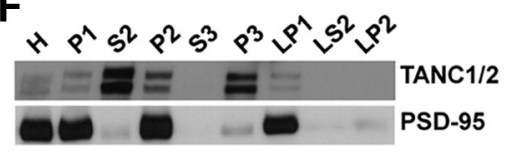

K

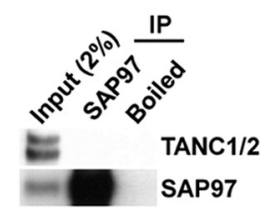

$\mathbf{L}$

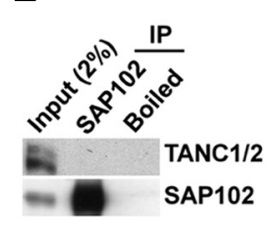

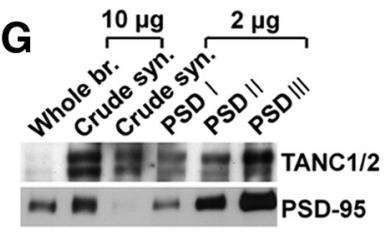

M

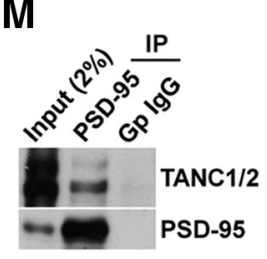

Figure 2. Expression patterns of TANC and in vivo association of TANC proteins with PSD-95. $\boldsymbol{A}$, Expression patterns of TANC1 and TANC2 mRNAs revealed by in situ hybridization in adult (6-week-old) rat brain sections. Ctx, Cortex; Cb, cerebellum; Hc, hippocampus; OB, olfactory bulb; St, stratum. Scale bar, 1 mm. $B$, Characterization of TANC antibodies. Synaptosomal (P2) and extrasynaptosomal (S2) rat brain fractions were immunoblotted with polyclonal pan-TANC (1609, rabbit) and TANC2-specific (1791, guinea pig) antibodies. $C$, Expression of TANC proteins in rat tissues. Sk. M, Skeletal muscle. D, TANC protein expression in rat brain regions. R., The other regions. $E$, Expression of TANC proteins during rat brain development. E, Embryonic stage; $P$, postnatal; W, week. $\boldsymbol{F}$, Localization of TANC proteins in subcellular fractions. H, Whole-brain homogenate; P1, whole homogenate pellet; S2, supernatant after P2 precipitation; P2, crude synaptosomes; S3, cytosol; P3, light membrane; LP1, synaptosomal membrane; LS2, synaptosomal cytosol; LP2, synaptic vesicle-enriched fraction. $\mathbf{G}$, Enrichment of TANC proteins in postsynaptic density (PSD) fractions. PSD fractions of adult rat brain extracted with Triton X-100 once (PSD I), twice (PSD II), or with Triton X-100 and the strong detergent sarcorsyl (PSD III) were immunoblotted with pan-TANC and PSD-95 antibodies. br., Brain; syn., synaptosomal (P2) fraction. H-L, TANC proteins form in vivo complexes with PSD-95 but not with other PSD-95 family proteins (PSD-93, SAP97, and SAP102). Deoxycholate extracts of the $P 2$ fraction of adult rat brains were immunoprecipitated with pan-TANC antibodies, boiled pan-TANC antibodies (negative control), or the indicated antibodies against PSD-95 family proteins, and analyzed by immunoblotting. $M$, TANC proteins coimmunoprecipitate with PSD-95 when the brain lysates removed of small membranes by high-speed centrifugation were used. RIPA extracts of the P2 fraction from adult rat brains were, high-speed centrifuged, immunoprecipitated with PSD-95 antibodies, and immunoblotted with pan-TANC and PSD-95 antibodies. Guinea pig $\lg G(\mathrm{Gp} \lg G)$ was used as a negative control antibody instead of boiled antibodies.

In yeast two-hybrid assays, TANC1 interacted with the PDZ domains from PSD-95 and PSD-95 relatives (PSD-93/chapsyn110 and SAP102), but not with other PDZ domains; these interactions were dependent on the C terminus of TANC1 (Fig. $1 B$ ). In addition, TANC1 and TANC2 formed a complex with PSD-95 in a manner that required the last three amino acid residues (Fig. 1C). In protein coclustering assays, COS cells doubly expressing PSD-95 and TANC2 formed discrete intracellular clusters where both proteins colocalized, whereas those expressing PSD-95 and a PSD-95 binding-deficient TANC2 mutant (TANC2 $\Delta$ C) did not (Fig. 1D). In GST pull-down assays using brain lysates, GST fusion proteins containing TANC1 and TANC2 C-terminal fragments, but not those that lacked PSD-95 binding (TANC1 $\Delta \mathrm{C}$ and TANC2 $\Delta$ C), pulled down PSD-95, PSD-93, SAP97 and SAP102, but not other PDZ domain proteins (GRIP1 and CASK) (Fig. 1E). These results suggest that PSD-95 interacts with TANC1/2 in vitro through PDZ domains.

We next examined the spine localization of TANC (WT and $\Delta C$ ) in cultured hippocampal neurons (DIV 18-19) doubly transfected with TANC1/2 (WT or $\Delta \mathrm{C}$ ) and PSD-95, which was used as a candidate PDZ protein to promote spine localization of TANC. Deletion of C-terminal residues from TANC1 or TANC2 significantly decreased their spine localization compared with their wild-type counterparts (Fig. $1 F, G$ ). In contrast, spine localization of PSD-95 protein was independent of TANC interaction. These results suggest that synaptic localization of TANC proteins requires their PDZ-binding $\mathrm{C}$ termini, which may be involved in the interaction with PSD-95 or some other PDZ proteins.

\section{Expression patterns of TANC and in vivo association of} TANC proteins with PSD-95

We determined the expression patterns of TANC1 and TANC2 mRNAs by in situ hybridization. TANC1 mRNAs were detected in various adult rat brain regions with particularly strong expression in hippocampus, thalamus, olfactory bulb, and cerebellum (Fig. 2A), similar to the previously described distribution patterns of TANC1 mRNAs (Suzuki et al., 2005). TANC2 mRNAs were also detected in various brain regions, but the signals were more evenly distributed throughout the brain, dissimilar to TANC1 mRNAs (Fig. 2A). 
We next generated polyclonal antibodies against TANC proteins using synthetic peptides mimicking parts of TANC1 and TANC2 sequences as immunogens. One antibody (designated 1609) recognized both TANC1 and TANC2 proteins and hence served as a pan-TANC antibody, whereas a second antibody (1791) was TANC2-specific (Fig. 2B). TANC1 and TANC2 proteins recognized by these antibodies had apparent molecular masses of $\sim 190$ and $\sim 210 \mathrm{kDa}$, respectively (Fig. 2 B). Although TANC1 mRNA is detected in a number of tissues (Suzuki et al., 2005), immunoblot analyses revealed that TANC1 and TANC2 proteins are mainly present in the brain (Fig. $2 C$ ), being detected in regions including the cortex, hippocampus, and cerebellum (Fig. 2D). Notably, TANC1 protein expression in the cerebellum was stronger than that of TANC2, in agreement with the strong in situ expression of TANC1 mRNA in the cerebellum (Fig. 2A). TANC1 expression increased during the first 1-2 weeks of postnatal rat brain development, whereas TANC2 was already highly expressed at embryonic day 18 and gradually decreased to adult levels beginning from the third postnatal week (Fig. 2 E). TANC1 and TANC2 proteins were detected in synaptic fractions, including crude synaptosomal (P2) and synaptic membrane (LP1) fractions (Fig. 2 F). Notably, however, a strong signal was also detected in the extra-crude synaptosomal (S2) and microsomal (P3) fractions, indicating that a significant portion of TANC proteins associate with small-vesicle membranes. Last, TANC1 and TANC2 proteins were enriched in postsynaptic density fractions; here, TANC2 was enriched to an extent similar to that of PSD-95 (Fig. $2 G$ ).

We also tested whether TANC proteins form in vivo complexes with PSD-95 family proteins. We found that both TANC1 and TANC2 proteins are present in complexes with PSD-95, but not with other PSD-95 relatives (PSD-93, SAP97, and SAP102) (Fig. $2 \mathrm{H}$ ). Immunoprecipitation in a reverse orientation yielded similar results (Fig. $2 I-L$ ). To more rigorously test the in vivo association between TANC and PSD-95, we repeated the immunoprecipitation experiment this time using brain detergent lysates removed of small vesicles by high-speed centrifugation. This experiment yielded the same results that TANC proteins associated with PSD-95 in vivo (Fig. $2 M$ ). In addition, the use of IgG instead of boiled antibodies as a control antibody gave the same results (Fig. $2 \mathrm{M}$ ). These results suggest that TANC proteins associate mainly with PSD-95 in vivo.

\section{TANC overexpression in cultured neurons increases the density of dendritic spines and excitatory synapses}

To explore possible functions of TANC proteins, we overexpressed TANC proteins in cultured hippocampal neurons and observed dendritic spines and excitatory synapses. Overexpressed TANC1 significantly increased the linear density of dendritic spines (Fig. $3 A, B$ ), without changing spine width or length (Fig. $3 A, C, D$ ). In addition, TANC1 overexpression increased the density of excitatory synapses, defined as PSD-95and synaptophysin-positive dendritic spines (Fig. 3E,F). The TANC1-dependent increases in spine and synapse densities required the C-terminal PDZ-binding motif, as shown by the absence of a spine-promoting effect in a TANC1 mutant that lacks the $\mathrm{C}$ terminus (TANC1 $\Delta \mathrm{C}$ ) (Fig. 3A,B). The absence of dominant-negative effects in TANC1 $\Delta \mathrm{C}$ might be attributable to insufficient levels of exogenous TANC1 $\Delta$ C expression relative to endogenous TANC1 levels. Overexpression of TANC2 in cultured neurons induced similar C terminusdependent, positive effects on the density of dendritic spines and excitatory synapses (Fig. $3 G-L$ ). These results suggest that
TANC1 and TANC2 regulate the density of dendritic spines and excitatory synapses.

\section{Generation of TANC1- and TANC2-deficient mice}

To further explore the functions of TANC1 and TANC2 proteins in vivo, we generated transgenic mice in which TANC1 or TANC2 was ablated using a gene-trap approach. Mouse ES cell lines containing a gene-trap cassette in TANC1 or TANC2 were used to generate the transgenic mice. The gene-trap cassette in TANC1 and TANC2 were localized to the introns between exons 17 and 18 , and exons 13 and 14, respectively, leading to the truncation of the proteins at the indicated positions (Fig. 4A,B). WT and gene-trap alleles were determined by PCR analysis (Fig. $4 C$ ). TANC1 $^{-1-}$ mice showed no detectable abnormalities at birth, and exhibited normal growth, body size, and breeding behavior. However, TANC2 ${ }^{-1-}$ mice died in utero, indicating that TANC2 is important for embryonic development.

TANC1 mRNA was undetectable in the brains of TANC1 ${ }^{-1-}$ mice, as revealed by in situ hybridization analysis of WT and TANC1 $^{-/-}$brain sections (Fig. $4 D$ ). TANC1 $^{-l-}$ mice did not express detectable levels of TANC1 proteins, whereas TANC2 protein levels were normal (Fig. $4 E$ ). Expression of other synaptic proteins, including PSD-95, were also unaffected by genetic ablation of TANC1 (Fig. $4 E$ ). The gross morphology of the TANC1 ${ }^{-/-}$brain was normal, as revealed by Nissl staining (Fig. $4 F$ ).

\section{TANC1 and TANC2 proteins exhibit distinct spatial distributions in the brain}

Using X-gal staining of gene-trapped TANC1- $\beta$-geo fusion proteins in TANC1 $1^{-1-}$ brain slices, which is a convenient way to determine the normal spatial expression pattern of the native protein, we detected TANC1- $\beta$-geo fusion proteins mainly in the hippocampus, thalamus, and cerebellum (Fig. $5 A$ ). In the hippocampus, the signals were strongest in the dentate gyrus (DG) and CA3 regions (Fig. $5 B$ ). In TANC2 ${ }^{+/-}$brain slices, X-gal staining was widely distributed in various brain regions, but was almost undetectable in the cerebellum (Fig. 5A). TANC2 signals in the hippocampus were mainly detected in the hilar region (Fig. $5 B)$. These results are consistent with the distribution patterns of TANC1 and TANC2 mRNAs (Fig. $2 A$ ), and suggest that TANC1 and TANC2 proteins are expressed in distinct brain regions. An important question would be whether these TANC1/2- $\beta$-geo fusion proteins have any residual functions, although fusion proteins are generally more prone to protein degradation, and these fusion proteins contain little or none of the ankyrin repeats that might have some functions. One way to address this question would be to determine the levels of the fusion proteins relative to full-length TANC proteins. However, we were unable to test this due to the lack of a suitable antibody against the $\mathrm{N}$-terminal portions of the proteins, which are present in both fusion and full-length products.

We next characterized spatial distribution patterns of TANC1 and TANC2 proteins in WT and TANC1 ${ }^{-1-}$ brain slices by immunohistochemistry. Immunostaining of WT slices with panTANC antibodies revealed the strongest signal in the CA3 region of the hippocampus, followed by the CA1 and DG regions (Fig. $5 C)$. These signals were mostly absent in the $\mathrm{TANC1}^{-1-}$ hippocampus stained with pan-TANC antibodies, which would allow only detection of TANC2 signals (Fig. $5 C$ ). The implication of these results - that the hippocampus expresses mainly TANC1 and not TANC2 - is in apparent contradiction with the immunoblot data shown in Figure $2 D$, which suggests that TANC1 and TANC2 are equally expressed in hippocampus. Because TANC2 
A

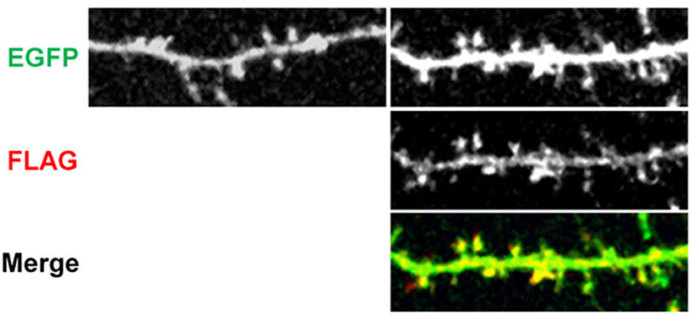

TANC1 $\Delta \mathrm{C}$

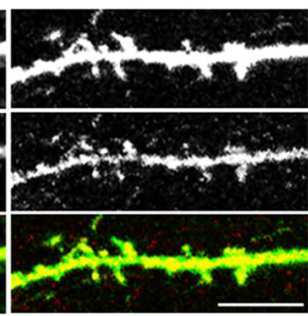

B

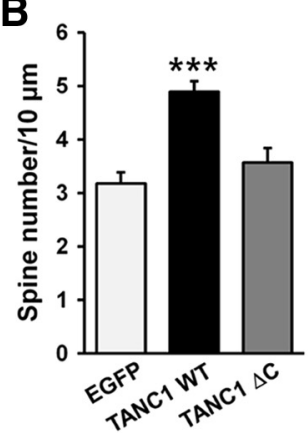

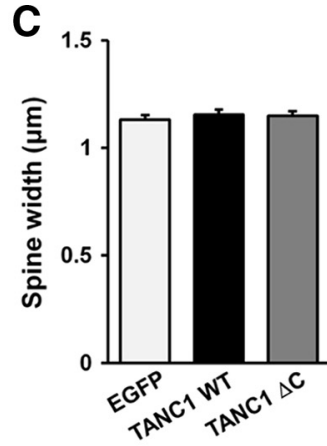

F
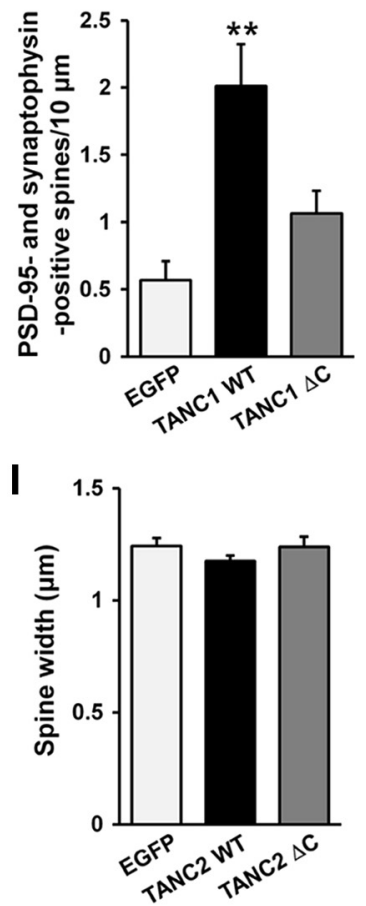

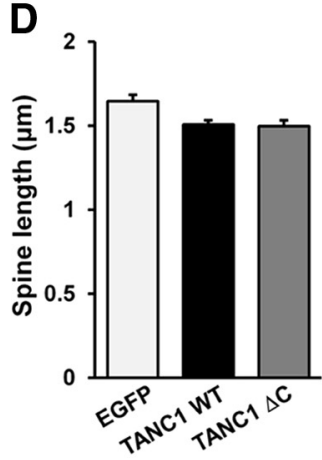

E

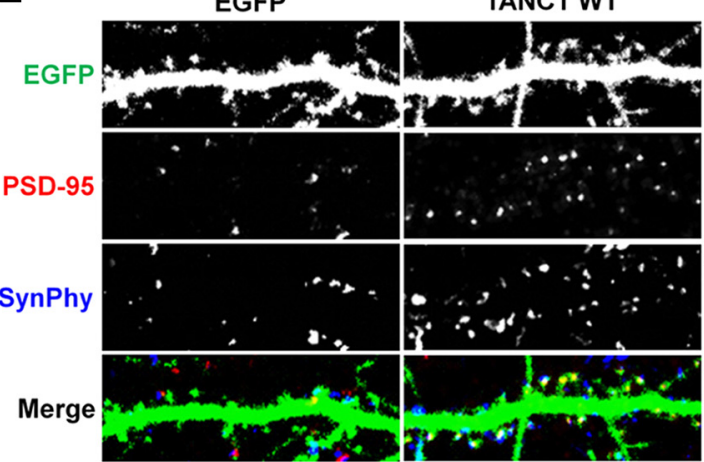

TANC1 $\Delta \mathrm{C}$

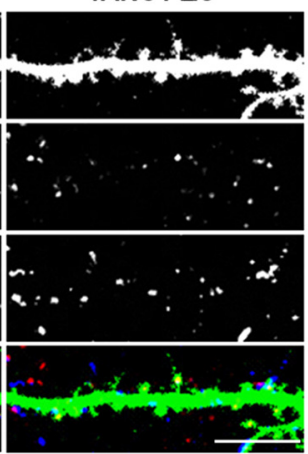

G

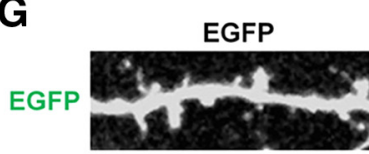

FLAG

Merge

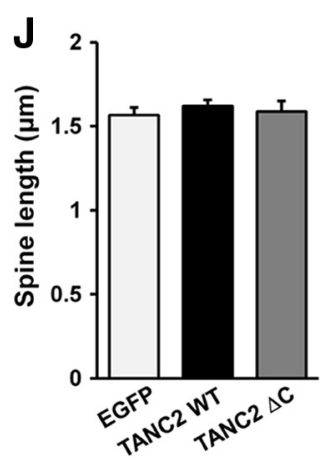

K

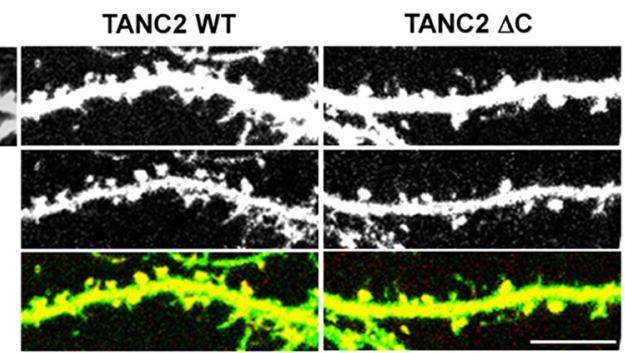

H

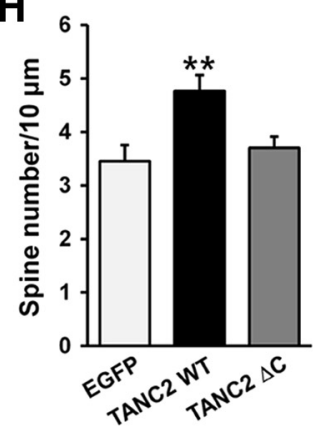

L

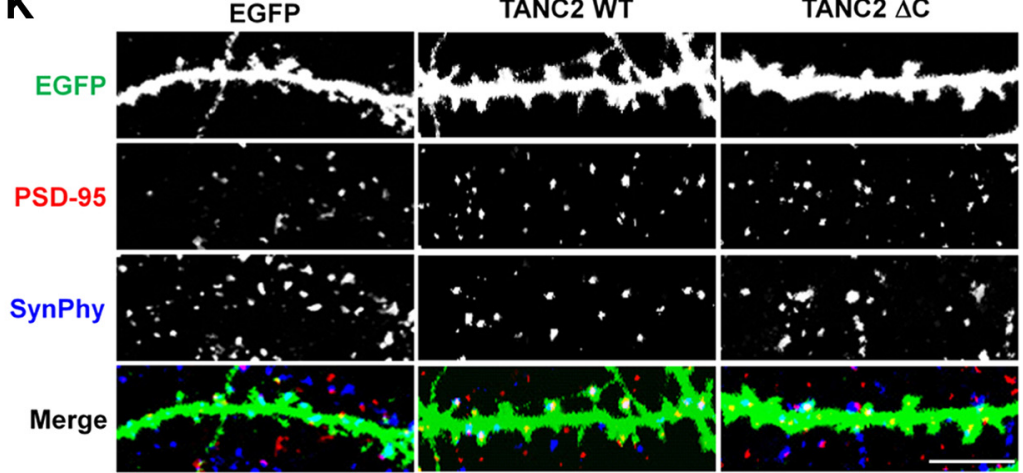

Figure 3. TANC overexpression in cultured neurons increases the density of dendritic spines and excitatory synapses. $A-D$, Overexpression of TANC1 increases the density, but not the width and length, of dendritic spines in a manner requiring PSD-95 binding. Cultured hippocampal neurons were transfected with FLAG-TANC1 (WT and $\triangle C$ ) and EGFP, or EGFP alone (DIV 8 -15), and stained for FLAG and EGFP. Dendritic spines were quantified for their linear density, head width, and length $(\boldsymbol{B}-\boldsymbol{D}) . n=29$ for EGFP alone control, 22 for WT, and 25 for $\Delta C,{ }^{* * *} p<0.001$, ANOVA and Tukey's test. $\boldsymbol{E}$, $\boldsymbol{F}$, TANC1 overexpression increases the density of excitatory synapses in a manner requiring the PDZ-binding C terminus. Transfected neurons were triply stained for EGFP, PSD-95, and synaptophysin (SynPhy). $n=12$ for control, WT, and $\Delta C,{ }^{* *} p<0.01$, ANOVA and Tukey's test. G-J, TANC2 overexpression increases spine density. $n=13$ for control, 17 for WT, and 15 for $\Delta C$, ** $p<0.01$, ANOVA and Tukey's test. $K$, $L$, TANC2 overexpression increases excitatory synapse density. Scale bars: $A, E, G, K, 10 \mu \mathrm{m} . n=18$ for control, 22 for WT, and 20 for $\Delta C$, ${ }^{* * *} p<0.001$, ANOVA and Tukey's test.

proteins are more tightly associated with the postsynaptic density (Fig. 2G), TANC2 proteins might have limited access to antibodies, making them difficult to visualize in the context of brain slices (unlike dissociated neurons), as previous reported for PSD-95 family proteins (Fukaya and Watanabe, 2000). However, staining of TANC1 ${ }^{-1-}$ slices with pan-TANC antibodies revealed relatively weak, but detectable, TANC2 signals in the subventricular zone (SVZ) of the hippocampus (Fig. 5D). Consistent with this, 
staining of WT slices with TANC2-specific antibodies also revealed detectable signals in the SVZ (Fig. 5E); similar TANC2 signals were also evident in TANC1 ${ }^{-/-}$hippocampus stained with TANC2 antibodies (Fig. $5 F$ ). The embryonic lethality of TANC2 ablation prevented us from performing similar experiments in TANC2 ${ }^{-1-}$ brain slices. Nonetheless, these results suggest that TANC1 and TANC2 proteins are expressed in distinct regions of the hippocampus.

We next determined the ultrastructural localization of TANC proteins in WT slices by electron microscopy. Pan-TANC antibodies revealed immunogold signals in dendritic spines and postsynaptic density in apical dendrites (stratum lucidum) of CA3 pyramidal neurons (Fig. 5G). Pan-TANC signals were also detected in various subcellular regions of neurons including the cell body, dendrites, and axon terminals (an example of an axon terminal is shown in Fig. 5G). We performed another type of EM analysis in which diaminobenzidine precipitation was used instead of immunogold particles to achieve a more sensitive quantification (supplemental Fig. S1, available at www.jneurosci.org as supplemental material). When we analyzed the distribution patterns of TANC signals in dendritic spines and axon terminals, the data indicated that $42.3 \pm 4.1 \%$ of dendritic spines contained TANC signals, while $13.4 \pm 1.3 \%$ of axon terminals were TANC positive (total $696.6 \mu \mathrm{m}^{2} ; n=3$ animals).

\section{Synaptic transmission and plasticity} are normal in the CA1 region of the TANC1 $^{-/-}$hippocampus

Because TANC1 was important for dendritic spines and excitatory synapses in cultured hippocampal neurons (Fig. 3), we tested whether TANC1 deficiency caused any changes in synaptic transmission or plasticity in the CA1 region of the hippocampus. The relationship between the amplitude of fiber volley and the slope of field EPSPs (inputoutput ratio) at synapses between CA3 Schaffer collaterals and CA1 pyramidal neurons (SC-CA1 synapses) was normal in TANC1 $^{-1-}$ mice (Fig. 6A), suggesting that AMPA receptormediated basal synaptic transmission was unchanged. The ratio of paired-pulse facilitation was also normal at $\mathrm{TANC1}^{-/-} \mathrm{SC}-$ CA1 synapses (Fig. $6 \mathrm{~B}$ ), suggesting that the probability of presynaptic release was unchanged. In addition, there were no changes in long-term potentiation induced by theta-burst stimulation (Fig. 6C), or long-term depression induced by low-frequency stimulation (Fig. 6D). These results suggest that TANC1 deficiency does not affect basal synaptic transmission or synaptic plasticity in the CA1 region of hippocampus.

Spine density is selectively decreased in the CA3 region of the TANC1 $^{-/-}$hippocampus

Although TANC1 deficiency had no effect on synaptic transmission or plasticity in the CA1 region of hippocampus (Fig. 6),
TANC1 protein is more strongly expressed in the CA3 region and, to a lesser extent, in the DG and CA1 regions of hippocampus (Fig. 5C). We thus tested whether TANC1 deficiency caused any changes in the dendritic spines of DG and CA3 regions, using DiOlistic delivery of lipophilic dyes (Gan et al., 2000). Interestingly, the spine density in the CA3 region of TANC1 ${ }^{-1-}$ hippocampus was significantly reduced, but was normal in the DG and CA1 regions (Fig. $7 A-C$ ). These results suggest that TANC1 is important for the maintenance of dendritic spines in the CA3 region of the hippocampus.

We next determined whether acute knockdown of TANC1 has a negative effect on excitatory synapses in cultured neurons. Transfection of cultured hippocampal neurons with a small interfering RNA (shRNA) construct targeting TANC1 (DIV 11/12-15/16) reduced the density of excitatory synapses, but it did not reach statistical significance, although the decreasing tendency could be rescued by an sh-RNA-resistant TANC1 expression construct (supplemental Fig. S2, available at www.jneurosci.org as supplemental material). One possible explanation for this result is that TANC1 is expressed at rela- 

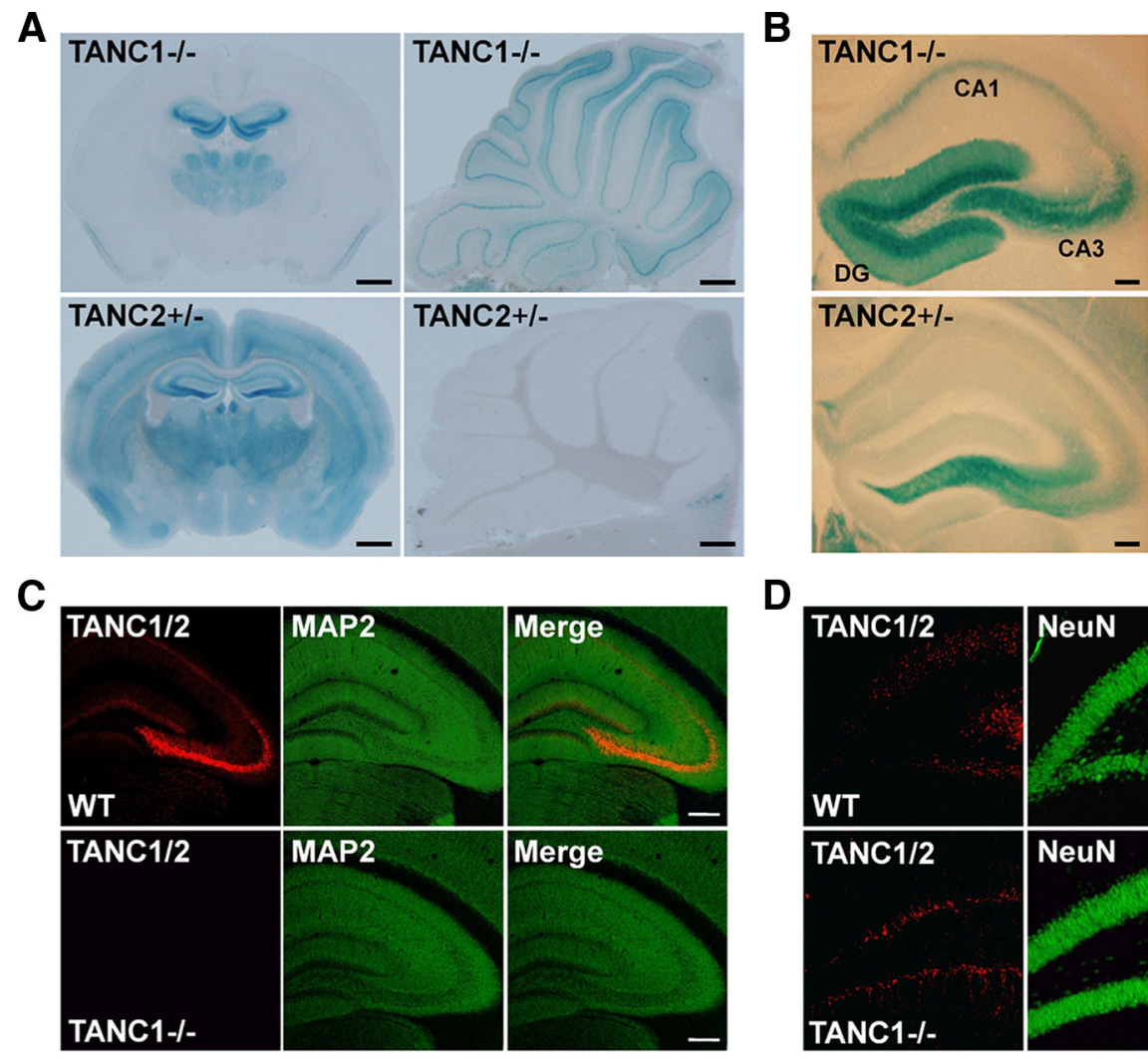

\section{D}

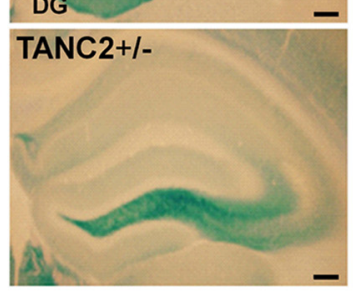

E
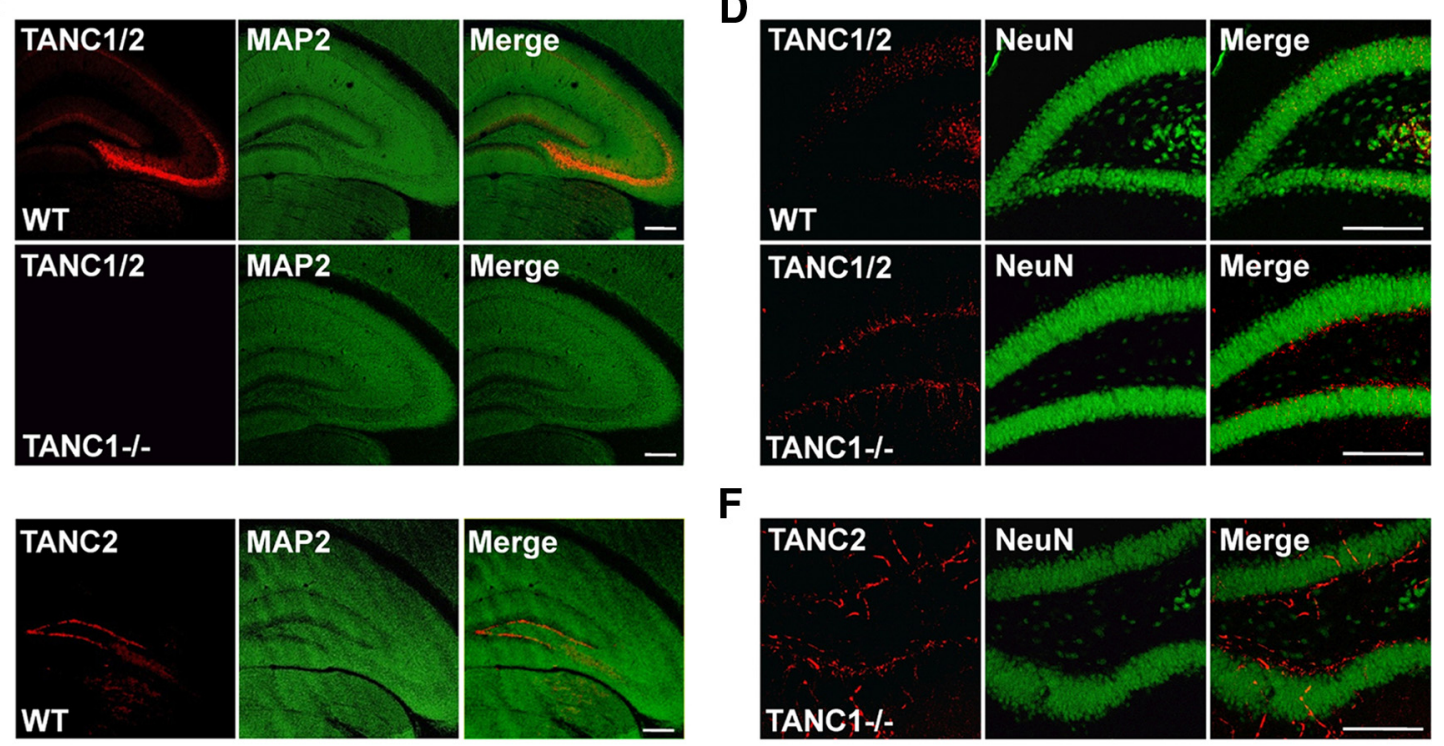

$\mathbf{F}$

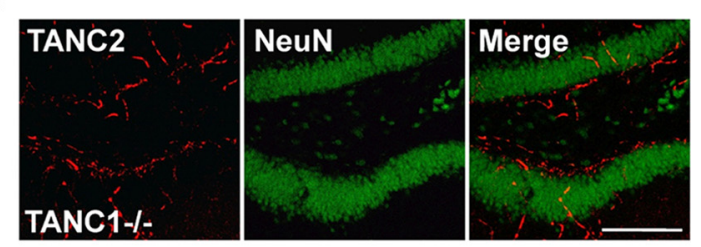

G
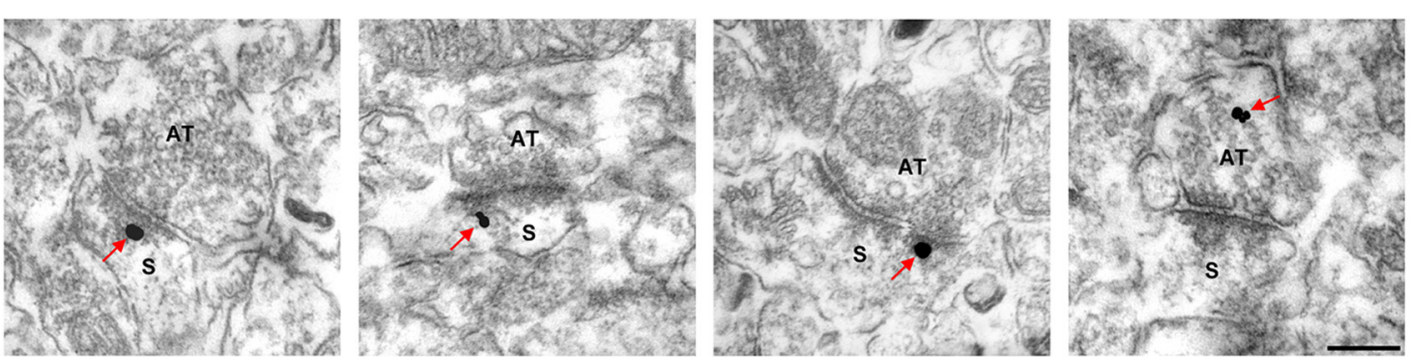

Figure 5. Distinct spatial distribution of TANC1 and TANC2 proteins revealed by $\beta$-gal and antibody staining of WT and TANC1 ${ }^{-1-}$ brain slices. $A, B$, Distinct expression patterns of TANC1 and TANC2 proteins in the whole brain $(\boldsymbol{A})$ and hippocampus $(\boldsymbol{B})$ revealed by $X$-gal staining of TANC1 ${ }^{-1-}$ and TANC2 ${ }^{+-}$brain slices. TANC2 ${ }^{-1-}$ slices could not be used for embryonic lethality. DG, Dentate gyrus. Scale bar, $250 \mu \mathrm{m}$. C, D, Spatial expression of TANC1 and TANC2 proteins revealed by staining of WT and TANC1 ${ }^{-1-}$ hippocampus with pan-TANC antibodies. Scale bar, $100 \mu \mathrm{m}$. $\boldsymbol{E}$, $F$, Spatial expression of TANC2 proteins revealed by staining of WT and TANC1 ${ }^{-1-}$ slices with TANC2-specific antibodies. Scale bar, $250 \mu \mathrm{m}$. G, Ultrastructural localization of TANC1 and TANC2 proteins in dendritic spines and the PSD revealed by preembedding immunogold electron microscopic staining of WT hippocampal slices with pan-TANC antibodies. The image shown here is from apical dendrites (stratum lucidum) in the hippocampal CA3 region. Red arrows indicate immunogold particles in dendritic spines (S; the first three images) and an axon terminal (AT; the last image). Scale bar, $0.2 \mu \mathrm{m}$.

tively high levels in CA3 pyramidal neurons, but only modestly in other neurons in DG and CA1 hippocampal regions.

Normal behaviors of $\mathrm{TANC}^{-1-}$ mice in open-field, elevated plus maze, and rotarod assays

For behavioral analysis, we first tested $\mathrm{TANC1}^{-/-}$mice in openfield assays. TANC1 ${ }^{-1-}$ mice showed normal levels of locomotor activity, movement speed, and time spent in the center zone (Fig. $8 A-C)$, suggesting that TANC1 deficiency does not affect exploratory activity, habituation, or anxiety-like behaviors. In the elevated plus maze, TANC1 ${ }^{-1-}$ mice spent normal amounts of time in open and closed arms, and showed a normal number of open-arm entries (Fig. $8 D, E$ ), further suggesting that $\mathrm{TANC}^{-l-}$ mice have normal levels of anxiety. In the rotarod test, $\mathrm{TANC1}^{-1-}$ mice showed latencies to fall from the rotating rod comparable to those in WT mice (Fig. $8 F$ ), suggesting that motor coordination in these mice is normal. These results suggest that TANC1 deficiency has minimal effects on exploratory behavior, anxiety-like behavior, and motor coordination. 
Impaired spatial memory in

TANC1 $^{-1-}$ mice

Finally, we tested whether TANC1 deficiency affects learning and memory behaviors. During the training phase of the Morris water maze (Morris, 1984), TANC1 ${ }^{-1-}$ mice learned to escape to the hidden platform to an extent similar to that of WT mice (Fig. 9A). In the probe test performed $24 \mathrm{~h}$ after the final training, TANC1 ${ }^{-1-}$ mice showed quadrant occupancies similar to those of WT mice (Fig. 9B). However, the number of exact crossings over a region where the platform was formerly located, a more stringent measure of spatial memory, was significantly reduced in $\mathrm{TANC1}^{-/-}$ mice (Fig. 9C). This result does not appear to be caused by reduced swimming speed of TANC1 $^{-1-}$ mice (Fig. 9D), or defects in sensory-motor function or motivation, as shown by the normal performance of $\mathrm{TANC1}^{-/-}$mice in the visible-platform version of the Morris water maze (Fig. 9E). These results suggest that TANC1 deficiency leads to a significant decrease in spatial memory.

\section{Discussion}

TANC2, a novel member of the TANC family of PSD-95-interacting proteins In the present study, we identified TANC2 as a novel member of the TANC family. TANC2 has features in common with those of the closely related TANC1; both are large proteins $(\sim 200 \mathrm{kDa})$ and contain the same protein-protein interaction domains. The C-terminal PDZ-binding motif of TANC2, like that of TANC1, mediates direct interaction with PSD-95 and is critical for localization of TANC2 to excitatory synapses. Functionally, both TANC1 and TANC2 have positive influences on dendritic spines and excitatory synapses in a manner that requires the PDZ-binding motif.

TANC2, however, has several features that are distinct from those of TANC1. TANC2 mRNA is widely distributed in various brain regions, whereas TANC1 mRNA is more concentrated in selected brain regions, including the hippocampus, thalamus, and cerebellum. Similarly, TANC1 protein expression, revealed by $\mathrm{X}$-gal staining of TANC1- $\beta$-geo fusion proteins, is mainly detected in the hippocampus, thalamus and cerebellum, whereas TANC2 protein is widespread, although the cerebellum shows an interesting absence of TANC2 protein. In subregions of the hippocampus, TANC1 protein is most abundant in CA3 pyramidal neurons, followed by the principal neurons in CA1 and DG regions. In contrast, TANC2 is more abundant in the subventricular zone, although the lack of TANC2 signals in principal neurons might reflect limited accessibility of anti-
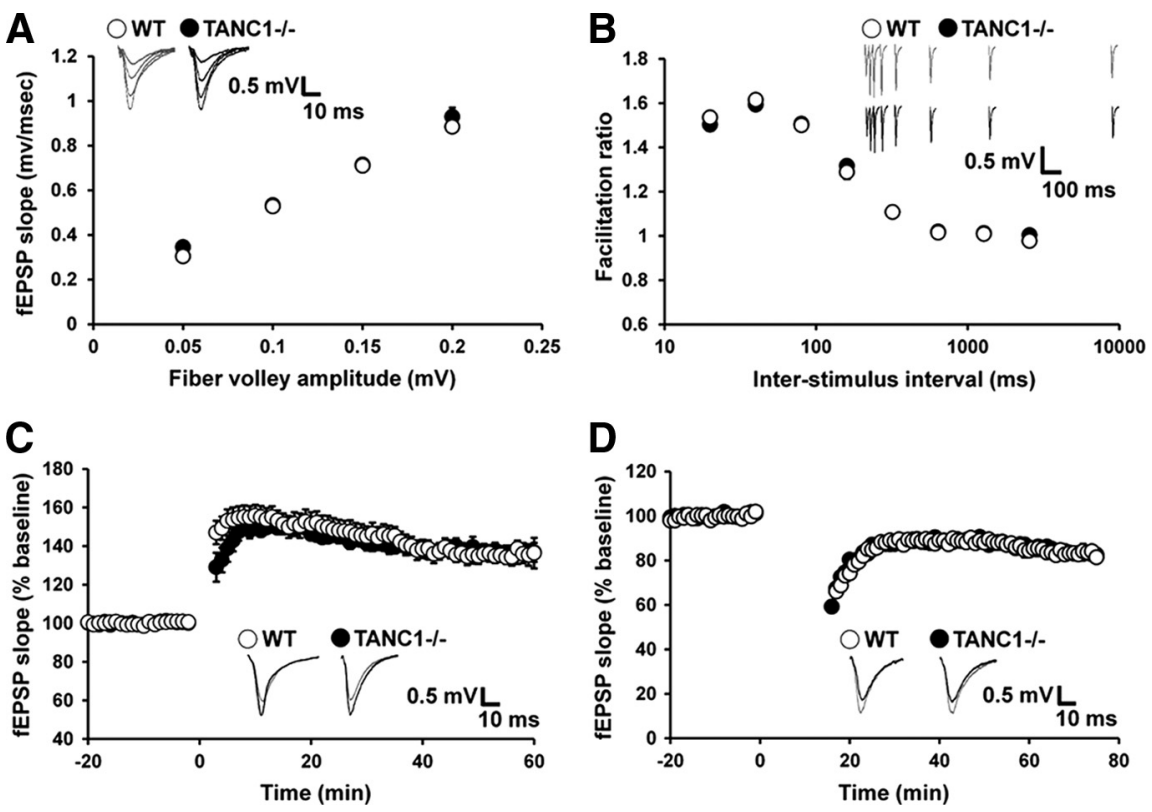

Figure 6. Normal synaptic transmission and plasticity in the CA1 region of TANC1 ${ }^{-/-}$hippocampus. $\boldsymbol{A}$, Normal basal transmission at TANC1 ${ }^{-1-}$ SC-CA1 synapses. The input- output relationship of AMPA receptor-mediated basal synaptic transmission was determined by plotting the slopes of field EPSPs (fEPSPs) against fiber-volley amplitudes. Sample traces of fEPSPs are shown in insets. WT, $n=10$ slices from 6 mice at 6 weeks; knock-out (KO), $n=17$ slices from 9 mice. $\boldsymbol{B}$, Normal paired-pulse facilitation ratio at TANC1 ${ }^{-1-}$ SC-CA1 synapses. Facilitation ratios were plotted against interstimulus intervals. WT, $n=14$ cells from 7 mice at 6 weeks; KO, $n=13$ slices from 5 mice. C, Normal LTP at TANC1 ${ }^{-1-}$ SC-CA1 synapses induced by theta burst stimulation. The gray and black traces were taken at 0 min and at the end of recording, respectively. WT, $n=10$ slices from 6 mice at 6 weeks; K0, $n=9$ slices from 6 mice. $D$, Unchanged LTD at TANC ${ }^{-1-}$ SC-CA1 synapses induced by low-frequency stimulation ( 900 paired pulses at $1 \mathrm{~Hz}$ ). WT, $n=13$ slices from 8 mice at 6 weeks; $K 0, n=20$ slices from 8 mice.
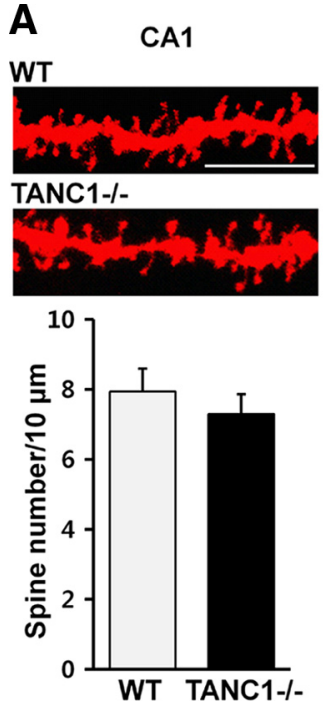

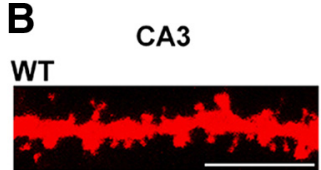

TANC1-I-
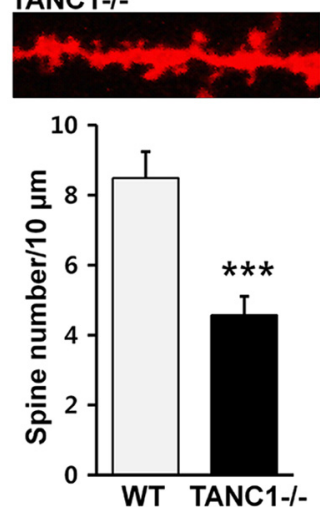

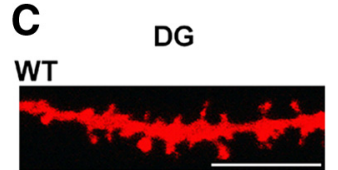

TANC1-/-
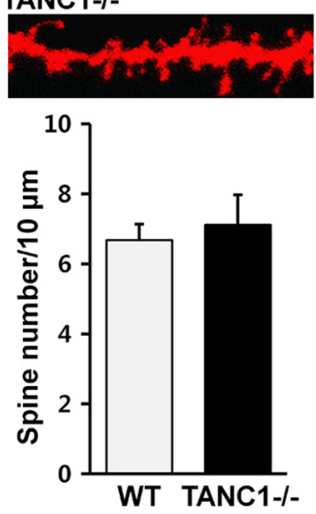

Figure 7. Selective decrease in spine density in the CA3 region of the TANC $1^{-/-}$hippocampus. $\boldsymbol{A}-\boldsymbol{C}$, Dendritic spines on apical dendrites of the principal neurons in CA1, CA3, and DG hippocampal regions (3 weeks) were visualized by DiOlistic delivery of Dil-coated particles, followed by quantification of spine density. Scale bar, $10 \mu \mathrm{m}$. CA1, $n=7$ neurons for WT and 8 for K0 from three pairs of mice; CA3, $n=14$ for WT and 11 for K0; DG, $n=9$ for WT and 6 for K0, ${ }^{* * *} p<0.001$, Student's $t$ test.

body staining reagents attributable to the tight association of TANC2 protein with the postsynaptic density. During rat brain development, TANC2 expression is detectable at embryonic stages and persists postnatally with a decreasing tendency, whereas TANC1 expression is initially low and gradually increases over time. Consistent with this, TANC2 deficiency is embryonic lethal, whereas TANC1 ${ }^{-1-}$ mice are normal at birth and show normal growth. 

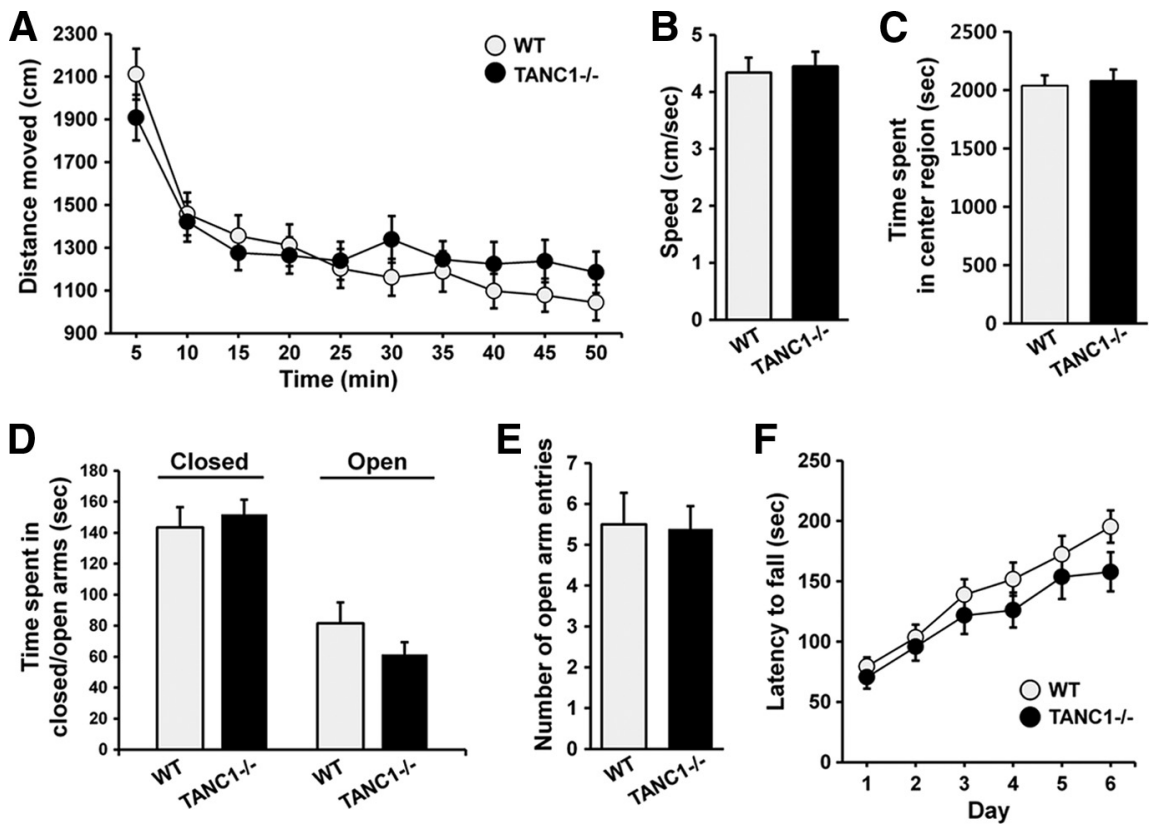

Figure 8. Normal behaviors of TANC1 ${ }^{-1-}$ mice in open-field, elevated plus maze, and rotarod assays. $\boldsymbol{A}_{-} \boldsymbol{C}$, TANC1 $^{-1-}$ mice show normal levels of total distance moved $(\boldsymbol{A})$, movement speed $(\boldsymbol{B})$, and time spent in the center region $(\boldsymbol{C})$ in open-field assays. $n=16$ for WT and 15 for KO (3-6 months). D, $\boldsymbol{E}$, TANC1 ${ }^{-1-}$ mice show normal amounts of time spent in closed/open arms (D), and a normal number of open arm entries $(\boldsymbol{E}) . n=16$ for WT and 15 for KO (3-6 months). $\boldsymbol{F}$, TANC1 ${ }^{-1-}$ mice show normal latencies to fall in rotarod assays. $n=16$ for WT and 15 for knock-out (3-6 months).
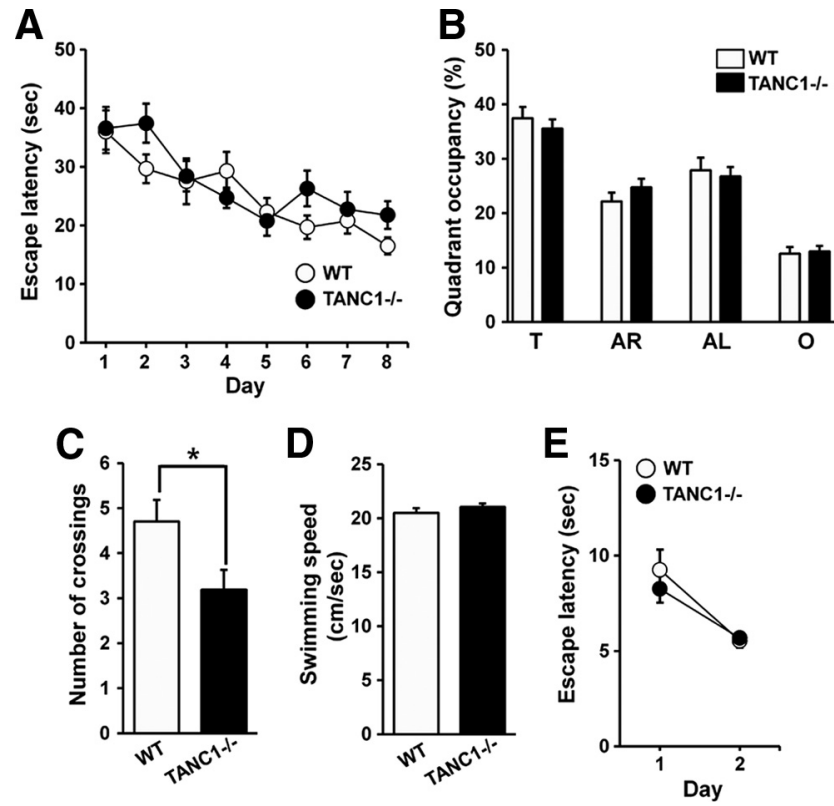

Figure 9. Impaired spatial memory of TANC1 ${ }^{-1-}$ mice in Morris water maze assays. $\boldsymbol{A}$, TANC1 $^{-1-}$ mice show normal levels of latency to find the hidden platform during the $8 \mathrm{~d}$ training phase. $n=20$ for WT and 21 for TANC1 ${ }^{-1-}\left(3-6\right.$ months). $B$, TANC $^{-1-}$ mice show levels of quadrant occupancies in the probe test comparable to those of WT mice. T, Target; AR, adjacent right; $A L$, adjacent left; 0 , opposite. $C$, TANC1 ${ }^{-1-}$ mice show a significantly reduced number of exact crossings over the region where the platform was formerly located during the probe test. $n=20$ for WT and 21 for TANC $1^{-1-},{ }^{*} p<0.05$, Student's $t$ test. $\boldsymbol{D}$, Normal swimming speed of TANC $1^{-1-}$ mice in the probe test. $\boldsymbol{E}$, Normal levels of learning in TANC1 $^{-l-}$ mice in the visible-platform version of the Morris water maze.

\section{Functions of TANC proteins}

Our results from TANC overexpression experiments indicate that TANC proteins are important for dendritic spines and excitatory synapses. This conclusion is further supported by the reduction in

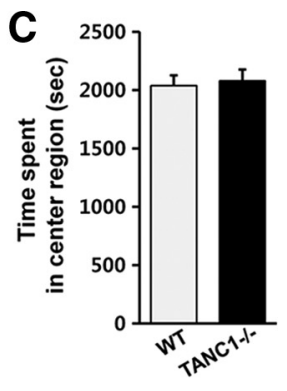

spine density in the hippocampal CA3 region of $\mathrm{TANC1}^{-/-}$mice. How might the maintenance of dendritic spines and excitatory synapses by TANC1 and TANC2 be regulated?

First, our results indicate that TANCdependent spine/synapse maintenance requires TANC binding to PSD-95, which promotes synaptic localization of TANC proteins. Thus, it is likely that interaction with PSD-95 concentrates TANC proteins at synapses, where they play a role in mediating PSD-95-dependent maintenance of spines and synapses. Second, from a functional perspective, synaptically translocated TANC proteins may function as a scaffold or adaptor for synaptic signaling by virtue of the presence of several domains for proteinprotein interactions, including ankyrin repeats, TPRs, a coiled-coil domain, and a C-terminal PDZ-binding motif. It should be noted, however, that a large portion of TANC proteins are present at extrasynaptic fractions. Some of them are likely to be dynamically translocated to excitatory synapses via PSD-95 interaction. In this context, a key determinant of synaptic localization of TANC would be the synaptic content of PSD-95. Interestingly, the synaptic localization of PSD-95 is reported to be regulated in an activity-dependent manner via mechanisms that include protein phosphorylation (Kim et al., 2007; Steiner et al., 2008). Whether PSD-95 is the only protein that promotes synaptic TANC localization is another point that needs to be addressed. The observation that TANC1 $\Delta \mathrm{C}$ and TANC2 $\Delta \mathrm{C}$ show reduced spine localization does not exclude the possibility that PDZ proteins other than PSD-95 may promote synaptic TANC localization.

Recently, it has been shown that the TPR and coiled-coil domains of TANC1 bind MINK and TNIK kinases (Nonaka et al., 2008). MINK was originally isolated as a gene whose expression is upregulated during postnatal mouse cerebral development (Dan et al., 2000), and both MINK and TNIK proteins are components of the postsynaptic density (Peng et al., 2004; Collins et al., 2006). MINK and TNIK are known to act as downstream effectors of Rap2 (Taira et al., 2004; Nonaka et al., 2008), which is a negative regulator of dendritic spines, excitatory synapses, and synaptic AMPA receptors (Pak et al., 2001; Pak and Sheng, 2003; Zhu et al., 2005; Fu et al., 2007; Ryu et al., 2008), similar to Rap1 (Zhu et al., 2002; Huang et al., 2004; Xie et al., 2005). Interestingly, MINK and TNIK promote TANC1 phosphorylation (Nonaka et al., 2008). These results, together with the TANC1-dependent positive regulation of dendritic spines and excitatory synapses, suggest the hypothesis that TANC1 phosphorylation by MINK/TNIK induced by Rap2 activation might result in inhibition of TANC1 and suppression of dendritic spines and excitatory synapses.

Clues for the functions of TANC proteins at the organismal level come from the phenotypes of TANC1 $1^{-1-}$ and $\mathrm{TANC}^{-1-}$ mice. TANC1 ${ }^{-1-}$ mice show reduced performance in the Morris water maze assay, while exhibiting normal behaviors in openfield, rotarod assays, and elevated plus maze. This suggests that TANC1 is important for spatial memory but not for locomotive exploration, motor coordination, or basal anxiety-like behaviors, although the involvement of TANC1 in learned anxiety such as 
fear conditioning remains to be determined. The fact that performance in the Morris water maze involves hippocampus-dependent spatial learning and memory suggests an association between the reduced spine density in the CA3 hippocampal region and impaired spatial memory in $\mathrm{TANC1}^{-/-}$mice.

In contrast to $\mathrm{TANC1}^{-/-}$mice, which survived to adulthood, TANC2 ${ }^{-1-}$ mice died in utero. We attempted to track the point at which embryonic death occurred, but could not detect $\mathrm{TANC}^{-1-}$ mice as early as embryonic day 12 , indicating that the embryos die before this time. Although further details remain to be elucidated, these results clearly indicate that TANC2 is important for early-stage embryonic development.

In conclusion, we found that TANC2, a novel member of the TANC family, has both unique features and features in common with TANC1. Both TANC proteins interact with PSD-95 and have positive influences on dendritic spines and excitatory synapses. This is further supported by the results from TANCdeficient mice, which additionally revealed distinct roles for TANC1 and TANC2 in spatial memory and embryonic development, respectively. Identifying the detailed mechanisms underlying these functions will be the focus of future studies.

\section{References}

Arnold DB, Clapham DE (1999) Molecular determinants for subcellular localization of PSD-95 with an interacting K+ channel. Neuron 23:149-157.

Cho KO, Hunt CA, Kennedy MB (1992) The rat brain postsynaptic density fraction contains a homolog of the Drosophila discs-large tumor suppressor protein. Neuron 9:929-942.

Choi J, Ko J, Park E, Lee JR, Yoon J, Lim S, Kim E (2002) Phosphorylation of stargazin by protein kinase A regulates its interaction with PSD-95. J Biol Chem 277:12359-12363.

Choi J, Ko J, Racz B, Burette A, Lee JR, Kim S, Na M, Lee HW, Kim K, Weinberg RJ, Kim E (2005) Regulation of dendritic spine morphogenesis by insulin receptor substrate 53 , a downstream effector of Racl and Cdc42 small GTPases. J Neurosci 25:869-879.

Collins MO, Husi H, Yu L, Brandon JM, Anderson CN, Blackstock WP, Choudhary JS, Grant SG (2006) Molecular characterization and comparison of the components and multiprotein complexes in the postsynaptic proteome. J Neurochem 97 [Suppl 1]:16-23.

Dan I, Watanabe NM, Kobayashi T, Yamashita-Suzuki K, Fukagaya Y, Kajikawa E, Kimura WK, Nakashima TM, Matsumoto K, Ninomiya-Tsuji J, Kusumi A (2000) Molecular cloning of MINK, a novel member of mammalian GCK family kinases, which is up-regulated during postnatal mouse cerebral development. FEBS Lett 469:19-23.

Fitzjohn SM, Doherty AJ, Collingridge GL (2006) Promiscuous interactions between AMPA-Rs and MAGUKs. Neuron 52:222-224.

Fu Z, Lee SH, Simonetta A, Hansen J, Sheng M, Pak DT (2007) Differential roles of Rap1 and Rap2 small GTPases in neurite retraction and synapse elimination in hippocampal spiny neurons. J Neurochem 100:118-131.

Fukaya M, Watanabe M (2000) Improved immunohistochemical detection of postsynaptically located PSD-95/SAP90 protein family by protease section pretreatment: a study in the adult mouse brain. J Comp Neurol 426:572-586.

Funke L, Dakoji S, Bredt DS (2005) Membrane-associated guanylate kinases regulate adhesion and plasticity at cell junctions. Annu Rev Biochem 74:219-245.

Gan WB, Grutzendler J, Wong WT, Wong RO, Lichtman JW (2000) Multicolor "DiOlistic" labeling of the nervous system using lipophilic dye combinations. Neuron 27:219-225.

Goslin K, Banker G (1991) Rat hippocampal neurons in low-density culture. In: Culturing nerve cells (Banker G, Goslin K, eds), pp 337-370. Cambridge, MA: MIT.

Han SB, Choi BI, Lee D, Kee SH, Kim HS, Sun W, Kim H (2009) Regulation of AHI1 expression in adult rat brain: Implication in hypothalamic feeding control. Biochem Biophys Res Commun 390:535-540.

Hsueh YP, Sheng M (1999) Regulated expression and subcellular localization of syndecan heparan sulfate proteoglycans and the syndecan-binding protein CASK/LIN-2 during rat brain development. J Neurosci 19:7415-7425.
Huang CC, You JL, Wu MY, Hsu KS (2004) Rap1-induced p38 mitogenactivated protein kinase activation facilitates AMPA receptor trafficking via the GDI. Rab5 complex. Potential role in (S)-3,5-dihydroxyphenylglyceneinduced long term depression. J Biol Chem 279:12286-12292.

Huttner WB, Schiebler W, Greengard P, De Camilli P (1983) Synapsin I (protein I), a nerve terminal-specific phosphoprotein. III. Its association with synaptic vesicles studied in a highly purified synaptic vesicle preparation. J Cell Biol 96:1374-1388.

Keith D, El-Husseini A (2008) Excitation control: balancing PSD-95 function at the synapse. Front Mol Neurosci 1:4.

Kim E, Niethammer M, Rothschild A, Jan YN, Sheng M (1995) Clustering of Shaker-type $\mathrm{K}+$ channels by interaction with a family of membraneassociated guanylate kinases. Nature 378:85-88.

Kim E, Cho KO, Rothschild A, Sheng M (1996) Heteromultimerization and NMDA receptor-clustering activity of Chapsyn-110, a member of the PSD-95 family of proteins. Neuron 17:103-113.

Kim MJ, Futai K, Jo J, Hayashi Y, Cho K, Sheng M (2007) Synaptic accumulation of PSD-95 and synaptic function regulated by phosphorylation of serine-295 of PSD-95. Neuron 56:488-502.

Menon SD, Chia W (2001) Drosophila rolling pebbles: a multidomain protein required for myoblast fusion that recruits D-Titin in response to the myoblast attractant Dumbfounded. Dev Cell 1:691-703.

Montgomery JM, Zamorano PL, Garner CC (2004) MAGUKs in synapse assembly and function: an emerging view. Cell Mol Life Sci 61:911-929.

Morris R (1984) Developments of a water-maze procedure for studying spatial learning in the rat. J Neurosci Methods 11:47-60.

Nonaka H, Takei K, Umikawa M, Oshiro M, Kuninaka K, Bayarjargal M, Asato T, Yamashiro Y, Uechi Y, Endo S, Suzuki T, Kariya K (2008) MINK is a Rap2 effector for phosphorylation of the postsynaptic scaffold protein TANC1. Biochem Biophys Res Commun 377:573-578.

Okabe S (2007) Molecular anatomy of the postsynaptic density. Mol Cell Neurosci 34:503-518.

Pak DT, Sheng M (2003) Targeted protein degradation and synapse remodeling by an inducible protein kinase. Science 302:1368-1373.

Pak DT, Yang S, Rudolph-Correia S, Kim E, Sheng M (2001) Regulation of dendritic spine morphology by SPAR, a PSD-95-associated RapGAP. Neuron 31:289-303.

Peng J, Kim MJ, Cheng D, Duong DM, Gygi SP, Sheng M (2004) Semiquantitative proteomic analysis of rat forebrain postsynaptic density fractions by mass spectrometry. J Biol Chem 279:21003-21011.

Rau A, Buttgereit D, Holz A, Fetter R, Doberstein SK, Paululat A, Staudt N, Skeath J, Michelson AM, Renkawitz-Pohl R (2001) rolling pebbles (rols) is required in Drosophila muscle precursors for recruitment of myoblasts for fusion. Development 128:5061-5073.

Ryu J, Futai K, Feliu M, Weinberg R, Sheng M (2008) Constitutively active Rap2 transgenic mice display fewer dendritic spines, reduced extracellular signal-regulated kinase signaling, enhanced long-term depression, and impaired spatial learning and fear extinction. J Neurosci 28:8178-8188.

Sheng M, Hoogenraad CC (2007) The postsynaptic architecture of excitatory synapses: a more quantitative view. Annu Rev Biochem 76:823-847.

Steiner P, Higley MJ, Xu W, Czervionke BL, Malenka RC, Sabatini BL (2008) Destabilization of the postsynaptic density by PSD-95 serine 73 phosphorylation inhibits spine growth and synaptic plasticity. Neuron 60:788-802.

Suzuki T, Li W, Zhang JP, Tian QB, Sakagami H, Usada N, Kondo H, Fujii T, Endo S (2005) A novel scaffold protein, TANC, possibly a rat homolog of Drosophila rolling pebbles (rols), forms a multiprotein complex with various postsynaptic density proteins. Eur J Neurosci 21:339-350.

Taira K, Umikawa M, Takei K, Myagmar BE, Shinzato M, Machida N, Uezato H, Nonaka S, Kariya K (2004) The Traf2- and Nck-interacting kinase as a putative effector of Rap2 to regulate actin cytoskeleton. J Biol Chem 279:49488-49496.

Xie Z, Huganir RL, Penzes P (2005) Activity-dependent dendritic spine structural plasticity is regulated by small GTPase Rap1 and its target AF-6. Neuron 48:605-618.

Zhu JJ, Qin Y, Zhao M, Van Aelst L, Malinow R (2002) Ras and Rap control AMPA receptor trafficking during synaptic plasticity. Cell 110:443-455.

Zhu Y, Pak D, Qin Y, McCormack SG, Kim MJ, Baumgart JP, Velamoor V, Auberson YP, Osten P, van Aelst L, Sheng M, Zhu JJ (2005) Rap2-JNK removes synaptic AMPA receptors during depotentiation. Neuron 46: 905-916. 